\title{
Manned Versus Unmanned Risk and Complexity Considerations for Future Midsized X-planes
}

\author{
Jason A. Lechniak ${ }^{1}$ \\ NASA Armstrong Flight Research Center, Edwards, California, 93523 \\ and \\ John E. Melton ${ }^{2}$ \\ NASA Ames Research Center, Moffet Field, California, 94035
}

\begin{abstract}
The objective of this work was to identify and estimate complexity and risks associated with the development and testing of new low-cost medium-scale $X$-plane aircraft primarily focused on air transport operations. Piloting modes that were evaluated for this task were manned, remotely piloted, and unmanned flight research programs. This analysis was conducted early in the data collection period for $\mathrm{X}$-plane concept vehicles before preliminary designs were complete. Over 50 different aircraft and system topics were used to evaluate the three piloting control modes. Expert group evaluations from a diverse set of pilots, engineers, and other experts at Aeronautics Research Mission Directorate centers within the National Aeronautics and Space Administration provided qualitative reasoning on the many issues surrounding the decisions regarding piloting modes. The group evaluations were numerically rated to evaluate each topic quantitatively and were used to provide independent criteria for vehicle complexity and risk. An Edwards Air Force Base instruction document was identified that emerged as a source of the effects found in our qualitative and quantitative data. The study showed that a manned aircraft was the best choice to align with test activities for transport aircraft flight research from a low-complexity and low-risk perspective. The study concluded that a manned aircraft option would minimize the risk and complexity to improve flight-test efficiency and bound the cost of the flight-test portion of the program. Several key findings and discriminators between the three modes are discussed in detail.
\end{abstract}

\section{Nomenclature}

$\begin{array}{ll}\text { 6-DOF } & =\text { six degrees-of-freedom } \\ \text { AFRC } & =\text { Armstrong Flight Research Center } \\ \text { ARMD } & =\text { Aeronautics Research Mission Directorate } \\ \text { ATC } & =\text { air traffic control } \\ \text { BLI } & =\text { boundary layer ingestion } \\ \text { CAS } & =\text { Convergent Aeronautics Solutions } \\ \text { DE } & =\text { development engineering } \\ \text { DFCS } & =\text { digital flight control system } \\ \text { EAFB } & =\text { Edwards Air Force Base } \\ \text { FAA } & =\text { Federal Aviation Administration } \\ \text { F-TS } & =\text { flight-test support } \\ \text { GVT } & =\text { ground vibration test } \\ \text { HILS } & =\text { hardware-in-the-loop simulation } \\ \text { NASA } & =\text { National Aeronautics and Space Administration } \\ \text { NRC } & =\text { National Research Council }\end{array}$

${ }^{1}$ Aerospace Engineer, Aerodynamics Branch, P.O. Box 273/MS 2228, Edwards, CA 93523, senior AIAA member.

${ }^{2}$ Aerospace Engineer, Systems Analysis Office, MS 258-1, Moffett Field, California, 94035, non-member. 


$\begin{array}{ll}\text { OR } & =\text { other requirements } \\ \text { RC } & =\text { research capability } \\ \text { RPV } & =\text { remotely piloted vehicle } \\ \text { TACP } & =\text { Transformative Aeronautics Concepts Program } \\ \text { UAS } & =\text { unmanned aircraft system } \\ \text { VR } & =\text { vehicle requirements } \\ \text { VS } & =\text { vehicle subsystems }\end{array}$

\section{Introduction}

$\mathrm{T}$ HE objective of this work was to provide a qualitative exploration of complexity and risk topics differentiating manned, remotely piloted, and unmanned X-plane concepts, and the operational challenges associated with each piloting control mode. This investigation was one of the first studies to be funded under a subproject in the Aeronautics Research Mission Directorate's (ARMD) new Convergent Aeronautics Solutions (CAS) project under the Transformative Aeronautics Concepts Program (TACP), which focuses on convergent solutions for future aeronautics research. This analysis was conducted before the ARMD's Spring 2016 announcement of a formal X-plane New Aviation Horizons Initative. ${ }^{1}$ Early task documentation encouraged an investigation of potential cost effective flight-test approaches to meet future ARMD flight research needs. Two near-term goals were also identified: 1) perform trade studies for vehicle configuration and concepts of operation and 2) develop vehicle research objectives and requirements. A National Research Council (NRC) report from 2012 also recommended that "NASA should also develop cost effective flight research vehicles to demonstrate innovative aerospace technology in flight." ${ }^{2}$ Ultimately, this work is intended to provide decision support data to estimate costs and risks associated with building and testing medium-sized X-plane aircraft, approximately the size of a Gulfstream Aerospace (Savannah, Georgia) GIII aircraft or larger. This paper describes the final approach chosen for the summary process, and provides some discussion of several interesting findings.

Three distinct modes of aircraft operation were compared during this study, and are defined as follows. The manned pilot control mode is defined as having at least one pilot on board the aircraft. The remotely piloted vehicle (RPV) control mode is defined as having at least one pilot in a ground control station that actively flies the aircraft in real time with no personnel on board the aircraft at any time. The autonomous pilot control mode is defined as having a preprogrammed flight path with control waypoints and at least one pilot at a ground control station actively monitoring the flight with the ability to alter the flight path for testing or emergency purposes. The autonomous pilot control mode also has no personnel on board the aircraft at any time.

The general framework of quantitative analysis ${ }^{3}$ was used to support evaluation of topics or themes. These topics and themes were identified and then assessed by an expert panel. The qualitative data alone are based on nonquantifiable topics surrounding research capabilities and vehicle requirements. A qualitative technique identifies, through subjective judgment, the important effects or influences of each proposed pilot control mode. The expert panel was composed of 22 members including manned and unmanned aircraft system (UAS) pilots, dynamics and control engineers, aeronautics systems analysts, operations engineers with UAS program experience, a UAS chief engineer, a cost estimator, aerodynamics engineers, a modeling and simulation engineer, and an instrumentation engineer. This group had a combined experience of over 400 man-years of aircraft experience. Due to the limited availablity of experts, time, and resources, the purposive/strategic ${ }^{3}$ sampling principle was used. The expert panel qualitatively identified, compared, and ranked a total of 54 different topic items using a simple quantitative scoring procedure. Additionally, the following quantitative method was used to help provide additional insight. A simplified rating system was developed using vehicle complexity and risk criteria. Numerical values from 0 to 5 were assigned to each topic, where a score of 0 indicated non-challenging influences, while a score of 5 indicated problematic influences.

Vehicle complexity encompasses a combination of the estimated schedule and costs. Risk criteria were a combination of both vehicle risk and mission risk. Vehicle risk and crew risk were assumed to have similar program consequences and were combined for the analysis. Some examples of vehicle risk resulting in loss of vehicle included loss of control and loss of situational awareness. Some examples of mission risk included immature technologies, integration difficulties, inability to collect research data, simulation limitations, and unknown unknowns.

Qualitative results of this study contain the major discussion points and high-level conclusions, while the quantitative results provide an estimate of the overall influence of the topic items. Both qualitative and quantitative results strongly favored a manned aircraft for transport aircraft flight physics research, based on estimated risk and complexity. 


\section{A. Candidate Aircraft Flight Research Goals}

A total of ten candidate aircraft configurations were initially considered by the CAS subproject for clean and efficient air transport X-planes. These ten configurations covered a broad spectrum of both classic aircraft configurations and more aerodynamically or structurally advanced configurations. Each configuration was considered for a large-scale flight research campaign similar to those of the X-29 (Grumman Aerospace Corporation, now Northrop Grumman Corporation, West Falls Church, Virginia) and X-31 aircraft (RockwellMesserschmitt-Bölkow-Blohm, United States / Germany), with about 100-150 flights. The configuration selection process was performed in parallel with this study, and focused on structural, aerodynamic, and propulsive issues, and the value of the flight research that each configuration could produce. The list of flight research goals and aircraft engineering issues (not prioritized and incomplete) was defined in early documentation and included:

1) Full-scale dynamics, handling qualities, and pilot workload impacts,

2) Boundary layer ingestion (BLI) propulsion system performance,

3) Test beds for multiple aerodynamic and propulsive technologies,

4) Low-speed stability and control performance,

5) Terminal area noise and acoustics analysis validation (engine shielding and airframe noise),

6) Flight controls for tailless vehicles,

7) Unique pressurization geometries,

8) Efficient transonic performance,

9) System integration,

10) Quantifiable structural efficiency gains.

\section{B. Expected Vehicle Specifications}

A wide range of vehicle factors made this evaluation difficult to bound and complicated the formulation of objective comparisons. Candidate vehicle physical configurations varied significantly in layout, size, and weight as determined by specific fight research objectives. Each aircraft was assumed to be about the same size as a Gulfstream GIII aircraft or larger. Approximate values assumed for the bounding physical configuration parameters were expected to exist in the following ranges:

1) Scale: $40 \%$ to $65 \%$ of full scale,

2) Wingspan: $55 \mathrm{ft}$ to $75 \mathrm{ft}$,

3) Empty weight: $14,000 \mathrm{lb}$ to $40,000 \mathrm{lb}$,

4) Primary Flight Condition: High subsonic to low transonic at altitudes from 20,000 ft to 35,000 ft MSL.

When combined with the flight-test goals listed in section A, these anticipated X-plane parameters yielded aircraft that would require testing at a large flight-test range. The most likely test location was identified as Edwards Air Force Base (EAFB) / National Aeronautics and Space Administration (NASA) Armstrong Flight Research Center (AFRC) (Edwards, California).

A detailed manned vs unmanned analysis of each concept vehicle with respect to the research and specifications could not be performed at the early stages of the analysis, as many of the configurations were conceptual at best. The scope of this study focused on the research and vehicle specifications from a more general perspective. A truly rigorous analysis and comparison would depend on the specifics of each vehicle configuration, requiring information on the level of a conceptual design for each. Given the wide variations in both the research goals and configurations of the ten candidate aircraft, this study focused instead on identifying complexity and risk characteristics that were thought to be common to any decisions regarding a future $\mathrm{X}$-plane.

\section{Range Limitations and Impact of Sense \& Avoid Requirements}

A small unmanned aircraft system (UAS) work area is available on the EAFB range. Autonomous and RPV vehicles that use the UAS work area can take off and land from the lakebed runways, and operations can be conducted on an unproven vehicle without significant impacts. Other EAFB aircraft are unaffected by unproven UAS operating in the UAS work area because the traffic patterns easily avoid the UAS work area. The work area, however, is constrained to RPV or autonomous vehicles with ceilings of $10,000 \mathrm{ft}$ or less travelling slower than 120 knots. At these maximum states, test conditions can only be held for short periods of time (typically about a few minutes in duration). The UAS work area would be too small for the aircraft and research objectives that were considered in this study.

Due to the task requirements of the target X-plane specification, the full range at EAFB would need to be utilized. The medium-sized UAS X-plane aircraft was projected to detect factor traffic cooperativly and would be unable to react to other air traffic quickly. In portions of the EAFB range the test altitude is unlimited, and in other portions the test ceiling can vary from $29,000 \mathrm{ft}$ to $50,000 \mathrm{ft}$. Test vehicles using the full range are subject to much stricter 
procedures and must be able to meet numerous operating constraints for safety and traffic avoidance. These limitations and constraints are documented in EAFB Instruction 13-100 ${ }^{4}$ which governs UAS flying and airfield operations.

UAS vehicles operating at EAFB are currently separated into five categories defined by their sense and avoid capabilities. The sense and avoid capability of the UAS is a risk reduction metric that impacts the airspace management of the test vehicle and the efficiency of flight-test operations. Chapter 14 of EAFB Instruction 13-100 4 governs UAS flying and airfield operations for UAS Operations. The items below (reprinted verbatim) provide a sense of the contraints on UAS operations and necessary equipment in the area of air traffic control (ATC). ${ }^{4}$

- 5 UAS type definitions segregate vehicles by sense and avoid and deviation capabilities:

- 14.4.1. Type 1: UAS has the ability to conduct sense and avoid to an equivalent level of capability as a manned aircraft (cooperative and non-cooperative traffic).

- 14.4.2. Type 2: UAS able to detect factor traffic (cooperative only) and take appropriate avoidance action in a timely manner (usually within a few seconds). The detection to action decision loop only involves the UAS and the operator.

- 14.4.3. Type 3: UAS able to detect factor traffic (cooperative only), but unable to react in a timely manner (usually within a few seconds). This delay may be due to detection method (ATC traffic monitoring, Chase aircraft) and/or latency inherent in UAS system (long link delays, complicated command sequences)

- 14.4.4. Type 4: UAS unable to deviate from flight path for traffic avoidance. ATC may be able to detect the conflict and direct the conflicting traffic to maneuver (ATC transponder required).

- 14.4.5. Type 5: UAS unable to deviate from flight path for traffic avoidance and ATC unable to accurately track a UAS to detect traffic conflicts (no transponder).

From the possible aircraft configurations under program consideration, "Type 3" was identified as the most probable designation. UAS risk mitigation requirements and procedures can also be found in Chapter 14 of EAFB Instruction 113-100 4 as well as RCC Document 323-99. ${ }^{5}$ A mitigation matrix provided therein outlines a general process that progresses risk mitigation from unproven (lowest level), through experimental (expected highest level for a medium-scale X-plane) to provisional and mature systems. An example of the required risk mitigation procedures for the unproven class of UAS is presented in Table 1 below.

Table 1. Common unproven class UAS mitigation requirements.

\begin{tabular}{|l|l|}
\hline \multicolumn{2}{|c|}{ Procedures } \\
\hline 1 & Exclusive use airspace \\
\hline 2 & Sanitized ground footprint \\
\hline 3 & Lakebed or exclusion zone takeoff and landing \\
\hline 4 & Chase aircraft to provide see and avoid \\
\hline 5 & Flight termination system \\
\hline 6 & Road closure \\
\hline
\end{tabular}

The RCC document 323-995 outlines range safety for unmanned air vehicles, which provides “...criteria based on guidance from safety specialists, existing reference standards and policies, and established procedures." This document establishes guidance for restricted airspace ranges and verifies compliance to National Airspace Federal Aviation Administration (FAA) requirements outside of restricted airspace. The document details the above criteria and indicates system maturity and system reliability serve as assurances that a UAS is safe to operate and will not pose additional risk to other vehicles, property, or human life. These assurances alone require significant restricted airspace operation to establish confidence in each airframe.

\section{Analysis Objectives and Assumptions}

An objective of this work was to provide decision support data and comparisons of the perceived risks of largescale X-plane concept vehicles with traceability to rationale and recommendations. Several separate studies done in parallel with this process showed a strong correlation between the cost and risk of building an airframe and the empty weight of the aircraft. ${ }^{6,7}$ Additionally, it was not expected that any high technical risk subsystems necessary for specific research objectives would be included in the airframe. The analysis was conducted assuming little

4

American Institute of Aeronautics and Astronautics 
technical risk up to the delivery of the airframe. In general, this study focused on the complexity and risk topics most prominent during the flight-testing portion of an X-plane program.

\section{A. Qualitative Analysis Objectives}

The qualitative analysis ${ }^{3}$ was expected to provide valuable information gained from the experience of flight-test engineers and staff. Detailed notes were kept to capture data used for the qualitative analysis. The data were used to provide a general understanding and to clarify and capture the complexities and risks associated with high level tradeoffs between manned and unmanned vehicles. Additionally, it was anticipated that the qualitative analysis would highlight several topics with strong supporting evaluation rationale.

\section{B. Quantitative Analysis Objectives}

The quantitative analysis was designed to include a general rating system intended to identify differences and similarities between each of the manned, remotely piloted, and unmanned X-plane piloting modes. The rating criteria for topic is outlined in Table 2. The authors attempted to use a time weighting criteria in addition to the analysis presented, but found that the time weighting had very little effect on the results and decided not to include the description or results. An expectation of the quantitative analysis was that it would identify impacts for each pilot control mode, which would in turn provide an overall result for the criteria as a whole.

Table 2. Topic rating criteria.

\begin{tabular}{|c|l|}
\hline $\begin{array}{c}\text { Rating } \\
\text { Value }\end{array}$ & \multicolumn{1}{c|}{$\begin{array}{c}\text { Rating Description } \\
\text { (Technical, Schedule, Cost) }\end{array}$} \\
\hline 5 & Very complex topic that was technical and/or contributed largely to cost and schedule \\
\hline 4 & Complex topic that was technical and/or contributed to cost and schedule \\
\hline 3 & Moderately complex topic that was somewhat technical and/or contributed to cost and schedule \\
\hline 2 & Mildly complex topic that was not very technical nor a considerable contribution to cost and schedule \\
\hline 1 & A requirement but not difficult to accomplish \\
\hline 0 & Not a requirement or not applicable \\
\hline
\end{tabular}

\section{Analysis Assumptions}

The X-plane concept vehicles were assumed to be subsonic medium-scale aircraft. Experience with testing this class of aircraft indicated that ejection seats would likely not be implimented in manned vehicles, and predetermined egress procedures could be executed in most emergency cases. In a program of this scale it was expected that the loss of vehicle would be equally detrimental in terms of risk as loss of a pilot; it was assumed that either would cause the program to be canceled. These assumptions are summarized in Table 3 below.

Table 3. Basic assumptions used for evaluation.

\begin{tabular}{|c|l|c|c|c|}
\hline \multicolumn{1}{|c|}{ Assumption } & Manned & RPV & Autonomous \\
\hline 1 & No ejection seat will be implemented on the test vehicle & $\checkmark$ & N/A & N/A \\
\hline 2 & Generalized evaluation of a medium-sized aircraft configuration & $\checkmark$ & $\checkmark$ & $\checkmark$ \\
\hline 3 & Risk to pilot and vehicle were not independently considered & $\checkmark$ & N/A & N/A \\
\hline 4 & Vehicle will be statically stable & $\checkmark$ & $\checkmark$ & $\checkmark$ \\
\hline 5 & Vehicle will operate subsonic and possibly transonic & $\checkmark$ & $\checkmark$ & $\checkmark$ \\
\hline 6 & Vehicle will be flown in the Edwards AFB range & $\checkmark$ & $\checkmark$ & $\checkmark$ \\
\hline 7 & Autonomous operation will not be a research objective & $\checkmark$ & $\checkmark$ & $\checkmark$ \\
\hline
\end{tabular}

The topic categories and data for this analysis were generated during multiple discussions with flight-test experts and pilots of both manned and unmanned aircraft at ARMD centers within NASA. In general, these topics and data were qualitative in nature regarding flight-testing functions. Table $4 \mathrm{a}$ presents the general themes and the distribution of complexity and risk topics within each theme. Table $4 \mathrm{~b}$ lists the specific 54 complexity and risk topics that were considered. Several of these topics were evaluated from different perspectives that included vehicle requirements (VR), research capability (RC), vehicle subsystems (VS), developmental engineering (DE), flight-test support (F-TS) and other requirements (OR). Several topics did not deviate over vehicle type. Some examples of these topics were transponders, radio communication, engine performance, and chase aircraft. 
Table 4a. Complexity and risk themes.

\begin{tabular}{|c|c|c|c|}
\hline \multicolumn{2}{|c|}{ Themes } \\
\hline Complexity & $\begin{array}{c}\text { Vehicle Requirements (VR) } \\
\text { 22 Topics }\end{array}$ & Research Capability (RC) & $\begin{array}{c}\text { Other Requirements (OR) } \\
17 \text { Topics }\end{array}$ \\
\hline \multirow{2}{*}{ Risk } & $\begin{array}{c}\text { Vehicle Subsystems (VS) } \\
\text { 20 Topics }\end{array}$ & $\begin{array}{c}\text { Developmental Engineering (DE) } \\
12 \text { Topics }\end{array}$ & $\begin{array}{c}\text { Flight-Test Support (F-TS) } \\
13 \text { Topics }\end{array}$ \\
\hline
\end{tabular}

Table 4b. Complexity and risk topics.

\begin{tabular}{|c|c|c|}
\hline \multicolumn{3}{|c|}{ Topics } \\
\hline Airframe noise (RC) & Flight envelope restrictions (OR) & Mission timeline (OR) (F-TS) \\
\hline Airframe performance (RC) (DE) & $\begin{array}{l}\text { Flight instrumentation and data } \\
\text { recording (VR) (VS) }\end{array}$ & Navigation (VR) (VS) \\
\hline Airspace availability (VS) & $\begin{array}{c}\text { Flight termination system } \\
\text { (VR) (VS) }\end{array}$ & $\begin{array}{c}\text { Operations workforce - Physical Danger } \\
\text { (OR) (F-TS) }\end{array}$ \\
\hline $\begin{array}{l}\text { Airworthiness process } \\
\text { (OR) (F-TS) }\end{array}$ & $\begin{array}{c}\text { Pilot display instrumentation (VR) } \\
\text { (VS) }\end{array}$ & $\begin{array}{c}\text { Operations workforce - Program } \\
\text { (OR) (F-TS) }\end{array}$ \\
\hline $\begin{array}{l}\text { Autopilot/Auto throttle/Auto land } \\
\text { (VR) (VS) }\end{array}$ & Flutter (RC) (DE) & Pilot situational awareness (VS) \\
\hline Chase aircraft (OR) (F-TS) & $\begin{array}{l}\text { Frequency requirements } \\
\text { (OR) (F-TS) }\end{array}$ & Pilot workload quantification (RC) \\
\hline $\begin{array}{c}\text { Cockpit/Ground control station } \\
\text { (VR) (VS) }\end{array}$ & Ground operation (OR) & Power requirements (VR) (VS) \\
\hline $\begin{array}{c}\text { Command and control link } \\
\text { (VR) (VS) }\end{array}$ & $\begin{array}{c}\text { Ground tests } \\
\text { - Preflight, GVT, etc. (OR) (DE) }\end{array}$ & Radar (VS) \\
\hline $\begin{array}{l}\text { Control law development } \\
\text { (RC) (DE) }\end{array}$ & $\begin{array}{c}\text { Ground tests } \\
\text { - Taxi, etc. (OR) (F-TS) }\end{array}$ & Radio communication (VR) (VS) \\
\hline Control room (OR) & Handling qualities (RC) (DE) & Range restrictions (OR) (F-TS) \\
\hline Egress (VR) (VS) & $\begin{array}{l}\text { Instrumentation ground testing } \\
\text { (F-TS) }\end{array}$ & Sense and avoid - airspace availability (VR) \\
\hline $\begin{array}{l}\text { Engineering workforce - } \\
\text { Developmental (OR) (DE) }\end{array}$ & Laminar flow (RC) (DE) & Sense and avoid - Pilot SA (VS) \\
\hline $\begin{array}{l}\text { Engineering workforce } \\
\text { - Test support (OR) (F-TS) }\end{array}$ & Line of sight (VR) (VS) & Simulation - HILS (OR) (DE) \\
\hline $\begin{array}{c}\text { Engine performance } \\
(\mathrm{RC})(\mathrm{DE})\end{array}$ & $\begin{array}{l}\text { Lost link - Mitigation } \\
\text { (VR) (VS) }\end{array}$ & Simulation - 6-DOF (OR) (DE) \\
\hline $\begin{array}{l}\text { Environmental control system } \\
\text { (VR) (VS) }\end{array}$ & $\begin{array}{c}\text { Lost link - Self Destruct } \\
(\mathrm{VR})\end{array}$ & $\begin{array}{c}\text { Stability and control - Development } \\
\text { (DE) (F-TS) }\end{array}$ \\
\hline $\begin{array}{l}\text { Envelope expansion } \\
\text { (OR) (F-TS) }\end{array}$ & $\begin{array}{l}\text { Mission management } \\
\text { (VR) (VS) }\end{array}$ & $\begin{array}{c}\text { Stability and control - Evaluation } \\
(\mathrm{RC})(\mathrm{VR})\end{array}$ \\
\hline $\begin{array}{l}\text { Flight control - Conventional } \\
\text { (VR) (VS) }\end{array}$ & Mission planning (OR) & Transponder (VR) (VS) \\
\hline $\begin{array}{c}\text { Flight control - Full DFCS } \\
\text { (VR) (VS) }\end{array}$ & Mission support (OR) (F-TS) & Upset recoveries (DE) \\
\hline
\end{tabular}

\section{Results}

The qualitative data for the complexity and risk topics were collected as experience-based observations or considerations that identified impacts and challenges specific to each of the piloting options. Scores for individual topics ranged from 0 to 5, with 0 assessed as low and 5 assessed as high complexity or risk. 


\section{A. Qualitative Complexity Results}

A large amount of experienced based analytical data were generated by the expert panel that identified support rational and challenges for each piloting mode of operation. This section summarizes the complexity results starting with the manned option, followed by the remotely piloted option and autonomous options.

\section{Manned Vehicle Results Synopsis}

Qualitative material that favored manned vehicles appeared in ground operations, instrumentation, and testing procedures. Ground operation restrictions were identified as minimal during flight-testing of manned vehicles relative to unmanned vehicles. The ground operations of new vehicles have numerous significant impacts due to Air Force directives. The manned vehicle option is largely unaffected by these directives. Key characteristics supporting the manned vehicle were:

1) A conventional control system could be implemented with no impact to the program.

2) Aircraft pilot display instrumentation requirements in a manned vehicle do not have to be complex to be functional.

3) For manned vehicles, the performance and flying qualities flight-testing procedures and processes are well known and would not require additional development.

Challenges for manned vehicles included cabin pressurization and emergency egress operations. Pressurization system requirements are expected to be more complex in manned vehicles as compared to unmanned vehicles. Depending on the configuration of the aircraft, emergency egress procedures would also be more complex. Although these challenges affect complexity and risk, elimination of each item was not expected to have a significant impact on the potential program. Manned vehicle operations are well known and regularly practiced, and tend to not increase flight-test complexity.

\section{Remotely Piloted Vehicle Results Synopsis}

Topics that favored remotely piloted vehicles included modeling, vehicle software assimilation, and enabling control law research. Similar software was expected to be used both for simulation and vehicle control where each would not be independent efforts, reducing superfluous duplication. Additionally, remotely controlled vehicles provide a platform that enables control law research.

Unfortunately, remotely piloted vehicles inherently encounter several challenging topics specific to flighttesting. Situational awareness of a remote piloted vehicle is limited and challenging, increasing the difficulty of many standard flight objectives and the complexity of vehicle recovery if needed. Typical processes were expected to require lost link risk mitigation with the ability to put the vehicle into a holding pattern until the link was restored and the implementation of a flight termination system in situations where a vehicle would become or was uncontrollable. These topics, together with uplink and downlink requirements, increase complexity both before and during flight-testing. Due to the above requirements, a digital flight control system would be highly recommended, further increasing complexity throughout the development, validation, and testing. The air and ground testing of unproven (Type 3) remotely piloted vehicles is also considerably more restricted than that of manned vehicles.

\section{Autonomous Vehicle Results Synopsis}

Supporting topics for unmanned autonomous vehicles included extended mission timelines, effective loss of signal mitigations, and enabling control law research. Similar to remotely piloted vehicles, autonomous vehicles provide a platform that enables control law research. Flight safety requirements for unmanned vehicles regarding loss of signal have proven and effective mitigations. Although not expected in any flight-testing of the proposed $\mathrm{X}$-planes, another supporting topic was the possibility of extended mission timelines (meaning tests longer than 8 hours).

Challenges that were identified regarding autonomous vehicles were the implementation of a flight termination system, uplink and downlink testing, and air and ground testing restrictions. These are the same challenges that were identified for the remotely piloted vehicles; however, additional challenges for fully autonomous operations were noted. An autonomous vehicle requires a digital flight control system that greatly increases complexity, development, validation, and testing. Emergency "non-standard" aborts are difficult to anticipate and perform in autonomous vehicles. A significant hurdle to efficient flight research was that mission management for fully autonomous operations often imposes multiple lengthy and complex preflight review processes. The survey group's collective experience with medium-sized autonomous vehicles indicated that flight-test programs conducted with vehicles that operate with full flight autonomy often become a project unto themselves, with costs and complexities that could be equal to or greater than the flight physics research of an X-plane program. 


\section{Complexity Results Summary}

All of the piloting modes shared some complexity topics, such as requirements of a chase aircraft during envelope expansion phases, which would include all if not most of the flight-test program. The chase aircraft requirement can be complex depending on scheduling, availabiliy, and matching capabilities of both aircraft. Other complexity topics that were shared among piloting modes were radio communication and power requirements.

Challenges that were captured for the manned X-plane vehicle were generally minor with relation to complexity. Expert observations identified numerous critical (but secondary) research topics that may be required before any flight research program can begin for remotely piloted and autonomous vehicle options. These secondary research topics were not explored further due to the large scope of potential X-plane vehicles. Some topics identified capabilities where remote piloting and autonomous vehicle options would be advantageous, but these capabilities did not support the task objectives. One example would be an extended mission timeline (longer than 8 hours) where having a pilot onboard could introduce prohibitive risk or complexity. For the task objectives, challenges for both the remote piloting and autonomous vehicle options increased complexity considerably. The conclusion from the qualitative results in this section strongly suggests that a manned X-plane vehicle is likely to be less complex overall than the other two piloting modes.

\section{B. Qualitative Risk Results}

Topic data were generated that identified supporting rational and challenges with respect to program and vehicle risk for each of the proposed types of vehicles. These topic data were then systematically scored in order to generate quantitative assessments.

\section{Manned Vehicle Results Summary}

Manned subsonic vehicles in the weight and class of the proposed vehicles have a long history of flight-test processes that are proven and established. Some of the possible new technologies to be investigated with the transport of X-planes, such as boundary layer ingestion engines or high aspect ratio truss-braced wings, are not common. In these cases a build-up approach to testing can be used to gradually push through the unknowns of the program. Other topics that were identified were common concerns regarding vehicle or pilot risk in an unknown vehicle configuration and emergency egress.

\section{Remotely and Autonomous Piloted Vehicle Results Summary}

Remotely piloted and unmanned vehicles were regarded as very similar with respect to program and vehicle risk for the following topics. Both types of vehicles would need a flight termination system to operate, range safety analysis, and associated mitigations for emergency situations, including loss of signal. Risks would include extremely difficult or challenging evaluation of handling qualities, limited upset maneuvering capability, and upset recovery testing. One of the most important aspects to these types of vehicles is that there is no risk to a pilot, copilot, or other required personnel.

Risks that were identified for remotely piloted vehicles were latency between command and response of the vehicle, command and force of the controls, and limiters that could restrict the aircraft from necessary recovery inputs in the event of an emergency.

Risks that were identified for autonomous vehicles were the inability to sufficiently test required systems for upset recovery; autopilot, auto throttle, and auto landing. Additionally, approach and landing abort logic would need to be integrated. The costs of some novel autonomous vehicles are sometimes further increased by necessary redundancies, as they require the construction and maintenance of both the aircraft and specialized ground stations as separate entities.

Common risks to both unmanned configurations were envisioned during the development and testing of digital flight control systems, associated human-readable instrumentation, and sense and avoid. There was also little confidence expressed in the notion of reusing or adapting ground and flight software from the existing mediumsized autonomous fleet.

\section{Risks Results Summary}

The general capability of the crew of a vehicle to complete sense and avoid detection and action was identified as a significant discriminating factor due to EAFB procedures. Most UAS are commonly considered to be too small for a pilot, limited to low speeds, inexpensive, and easily replaceable while presenting low risk to the uninvolved public. While the complete loss of a small UAS would likely have relatively minimal concequences to a program, the same can not be said for medium and large UAS. The development and test schedules of larger, faster UAS systems are often extended for a variety of schedule and safety reasons. As a best practice, an effect of Instruction 
113-1004 and RCC Document 323-99, ${ }^{5}$ UAS versions of the X-plane aircraft would be considered unproven for at least the first five flights. The unproven designation takes into consideration risks to the nearby population, workforce, and high value assets. Early flights are often restricted to sanitized airspace on weekend-only (Saturday) days. Progression to the experimental risk mitigation level would relieve some of the restrictions in Table 1. Other issues include cancellations, unforeseen failures, and modifications. Flight-test cancellations are common due to weather, instrumentation failures, software, early development problems, and many other issues. Modifications such as software updates can be expected to demote or reclassify the vehicle back down into the unproven category, requiring the repeat of previous qualification flights. Due to all of the dependencies described, it is inevitable that the flight schedules (and flight-test campaigns) of UAS X-planes will be impacted and significantly extended. As a consequence of the directions outlined in Chapter 14 of EAFB 13-1004 instructions and RCC Document 323-99, ${ }^{5}$ the autonomous functions of both types of unmanned aircraft become a significant part of the flight test, which threatens both the budget and schedule scope for flight-test research.

\section{Quantitative Results}

Quantitative values for each topic were assessed in discussions with experts and vetted through group consensus. These criteria (Table 2) were assigned a value from 0 to 5 , with 0 indicating low and 5 indicating high complexity or risk.

For some of the topics, assigning quantitative values was straightforward. For example, the implementation of classical flight instruments for a manned vehicle is comparatively simple and therefore assigned a quantitative value of one (1). Conversely, assigning quantitative values was quite difficult for other topics. Qualitatively, the implementation of environmental control systems for an RPV or autonomous vehicle appeared to be a simple evaluation. Environmental control systems for an RPV or autonomous vehicle (such as heating, cooling, and weather shielding) were identified as an early requirement, but assigning a consensus quantitative score during group review was difficult.

Summaries of the quantitative results are presented below. The complete list of complexity topics is included in Appendix A, while Appendix B contains the list of risk topics.

\section{Complexity Results}

A summary of the quantitative complexity results is shown in Table 5. Applicable complexity topics from Table $4 \mathrm{~b}$ were itemized under one of the three categories: VR, RC, and OR. In all of the categories, the manned operation mode consistently scored approximately half as complex as either the RPV or autonomous modes. The quantitative complexity results numerically support the findings of the qualitative results.

Table 5. Quantitative complexity results.

\begin{tabular}{|c|c|c|c|c|c|c|}
\hline \multicolumn{7}{|c|}{ Complexity } \\
\hline $\begin{array}{c}\text { Vehicle Requirements (VR) } \\
\text { 22 Topics }\end{array}$ & Manned & $\%$ & RPV & $\%$ & Autonomous & $\%$ \\
\hline $\begin{array}{c}\text { Research Capability (RC) } \\
\text { 9 Topics }\end{array}$ & 27 & 19.4 & 52 & 37.4 & 60 & 43.2 \\
\hline $\begin{array}{c}\text { Other Requirements (OR) } \\
\text { 17 Topics }\end{array}$ & 36 & 22.0 & 62 & 37.8 & 66 & 40.2 \\
\hline Total (48 Topics) & 84 & $\mathbf{2 1 . 5}$ & 145 & $\mathbf{3 7 . 1}$ & 162 & $\mathbf{4 1 . 4}$ \\
\hline
\end{tabular}

Each of the topics from Table $4 \mathrm{~b}$ were ranked using values from 0 to 5 . The complexity scores for each topic were separated into three tiers; a score of 0 or 1 was assigned a green bar that indicated low-challenge influences, a score of 2 or 3 was assigned a yellow bar that indicated medium challenge, and a score of 4 and 5 was assigned a red bar that indicated highly challenging influences.

Table 6 contains the topics with the largest deviations in complexity. These topics were identified to have both low and highly challenging influences depending on which piloting mode is chosen. The only topic identified as highly challenging for a manned vehicle when compared to RPV and autonomous was the egress topic. Table 6 also indicates that the RPV mode was expected to lie between manned and autonomous in terms of complexity. A total of 20 topics were expected to be medium to highly challenging for RPV and autonomous vehicles, with autonomous operations expected to be the most complex. These complexity results in Table 6 further support the findings of the qualitative and quantitative results in Table 5. 
Table 6. Complexity topics with the largest deviation.

\begin{tabular}{|c|c|c|c|c|c|}
\hline Complexity & Manned & RPV & Autonomous & Total & St Dev \\
\hline Line of sight & 0 & 3 & 2 & 5 & 1.25 \\
\hline Flight termination system & 0 & 3 & 3 & 6 & 1.41 \\
\hline Sense and avoid - Pilot SA & 1 & 2 & 3 & 6 & 1.25 \\
\hline Stability and control (VR) & 1 & 1 & 4 & 6 & 1.41 \\
\hline Egress & 4 & 1 & 1 & 6 & 1.41 \\
\hline Mission management & 1 & 2 & 4 & 7 & 1.25 \\
\hline Command and control link & 0 & 4 & 3 & 7 & 1.70 \\
\hline Lost link - Mitigation & 0 & 4 & 3 & 7 & 1.70 \\
\hline Cockpit/Ground control station & 1 & 4 & 3 & 8 & 1.25 \\
\hline Autopilot/Auto throttle/Auto land & 1 & 2 & 5 & 8 & 1.70 \\
\hline Ground operation & 1 & 3 & 4 & 8 & 1.25 \\
\hline Flight control - conventional & 1 & 3 & 4 & 8 & 1.25 \\
\hline Mission planning & 1 & 3 & 4 & 8 & 1.25 \\
\hline Pilot workload quantification & 1 & 3 & 5 & 9 & 1.63 \\
\hline Handling qualities & 1 & 4 & 5 & 10 & 1.70 \\
\hline Simulation - HILS & 2 & 3 & 5 & 10 & 1.25 \\
\hline Envelope expansion & 2 & 4 & 5 & 11 & 1.25 \\
\hline Pilot display instrumentation & 2 & 4 & 5 & 11 & 1.25 \\
\hline Flight envelope restrictions & 2 & 4 & 5 & 11 & 1.25 \\
\hline Operations workforce & 2 & 5 & 5 & 12 & 1.41 \\
\hline
\end{tabular}

Each of the topics was then itemized into categories indicating overall low to high challenge, as shown in Appendix A. In general, the trend of Appendix A suggests the RPV and autonomous piloting modes of operation are expected to be more complex. Appendix A could also be useful for other purposes as it identifies low-, medium- and highly-challenging topics for the overall program independently of the piloting mode of operation.

\section{Risk Results}

A summary of the quantitative risk results is shown in Table 7. Applicable risk criteria from Table 2 were itemized under one of the three categories: VS, DE, and F-TS. In the vehicle subsystems category the manned operation mode was estimated to have approximately half the risk of either the RPV or autonomous modes. The RPV operation mode risk was evaluated to be significantly less than the autonomous operation mode in many individual categories. The manned operation mode was identified to contain half the risk of the autonomous modes in many individual categories. The quantitative risk results numerically support the findings of the qualitative risk results.

Table 7. Quantitative risk results.

\begin{tabular}{|c|c|c|c|c|c|c|}
\hline \multicolumn{7}{|c|}{ Risk } \\
\hline $\begin{array}{c}\text { Vehicle Subsystems (VS) } \\
\text { 20 Topics }\end{array}$ & Manned & $\%$ & RPV & $\%$ & Autonomous & $\%$ \\
\hline $\begin{array}{c}\text { Developmental Engineering } \\
\text { (DE) } \\
12 \text { Topics }\end{array}$ & 22 & 25.6 & 28 & 32.6 & 36 & 41.9 \\
\hline $\begin{array}{c}\text { Flight-Test Support (F-TS) } \\
13 \text { Topics }\end{array}$ & 23 & 23.5 & 35 & 35.7 & 40 & 40.8 \\
\hline Total (45 Topics) & 72 & $\mathbf{2 2 . 4}$ & 118 & $\mathbf{3 6 . 8}$ & 131 & $\mathbf{4 0 . 8}$ \\
\hline
\end{tabular}

Each of the risk topics were also ranked using values from 0 to 5 . The scores were separated as described in the section III.C.1.

Table 8 presents the risk challenges that showed a strong dependency with the piloting mode of operation. Nine topics were expected to be more challenging for RPV and autonomous vehicles, again showing a trend that each of 
these piloting modes of operation are expected to be more difficult. The complexity results in Table 8 further support the findings of the qualitative results and numerical results.

Table 8. Risk topics with the largest deviation.

\begin{tabular}{|c|c|c|c|c|c|}
\hline Risk & Manned & RPV & Autonomous & Result & St Dev \\
\hline Lost link & 1 & 4 & 2 & 7 & 1.25 \\
\hline Airframe performance & 2 & 1 & 4 & 7 & 1.25 \\
\hline Pilot situational awareness & 1 & 3 & 4 & 8 & 1.25 \\
\hline Command and control link & 1 & 4 & 3 & 8 & 1.25 \\
\hline Cockpit/Ground control station & 1 & 5 & 3 & 9 & 1.63 \\
\hline Autopilot/Auto throttle/Auto land & 1 & 4 & 4 & 9 & 1.41 \\
\hline Airworthiness process & 2 & 3 & 5 & 10 & 1.25 \\
\hline Flight control - Conventional & 1 & 4 & 5 & 10 & 1.70 \\
\hline Handling qualities & 1 & 4 & 5 & 10 & 1.70 \\
\hline Mission Timeline & 1 & 4 & 5 & 10 & 1.70 \\
\hline
\end{tabular}

As stated in section III.C.1, each of the topics was itemized into categories indicating overall low to high risk, and are included in Appendix B. In general, there is a strong trend in Table 8 that suggests the RPV and autonomous piloting modes of operation are expected to have more risk. Appendix B could also be useful for other purposes, as it identifies low-, medium- and high-risk topics for the overall program independently of the piloting mode of operation.

An attempt to time weight the results in Table 5 and Table 7 produced small differences in the overall results and is not presented.

\section{Conclusion}

The aircraft studied during the CAS X-plane subproject were chosen to demonstrate new approaches for revolutionary increases in sustainable air transportation flight efficiency. All of the CAS X-plane study vehicles considered were medium-sized vehicles, approximately the size of a G-III aircraft, with the ability to sustain flight conditions appropriate for subsonic and transonic air transport research operations. Analysis assumptions were summarized in Table 3. Additionally, the X-plane concept vehicles were assumed to be subsonic medium-scale aircraft. In a program of the projected scale, the loss of vehicle was anticipated to be equally detrimental in terms of risk as loss of a pilot; it was assumed that either would cause the program to be canceled.

Expert group evaluations provided qualitative reasoning and substance from a diverse set of pilots, engineers, and other experts on the many issues surrounding the decisions about piloting options. The group evaluations were estimated within each topic to provide a quantitative result that summarizes and supports the qualitative results.

An EAFB instruction document was identified that, when implemented, mitigates risk by requiring a new or low flight number vehicle to systematically perform sorties and pass numerous review boards during the testing of the vehicle before being granted further operational flexibilities. The EAFB document clearly describes the accepted maturation process and specifies the extra restrictions applied to unmanned vehicles throughout the various developmental and testing stages. The EAFB instruction $13-100^{4}$ as well as the implications of its implementation emerged as the source of the effects found in the qualitative and quantitative data.

Overall, this study concluded that a manned aircraft option could be expected to suppress complexity and risk for a potential X-plane program as defined in this document. The reduction of these factors was expected to enhance flight research efficiency and constrain the cost of the flight-test portion of the program. Autonomous functions in UAS vehicles have a way of becoming a large part of the test program, which would likely distract from the fundamental flight experiment and/or increase costs and schedule. In demonstration of the primary research objective, air transportation flight physics, both remotely piloted and fully autonomous vehicles are likely to increase both cost and schedule compared to conventional manned operations. The study showed both qualitatively and quantitatively that a manned aircraft was the best choice to align with test activities for transport aircraft flight research from a reduced complexity and low risk perspective. 
Appendix A: Summarized Results for all Complexity Topics

\begin{tabular}{|c|c|c|c|c|c|c|}
\hline Complexity & Manned & RPV & Autonomous & Total & St Dev & Result \\
\hline Lost link - Self-destruct & $\mathbf{0}$ & 1 & 1 & 2 & 0.47 & \multirow{5}{*}{$\begin{array}{l}\text { Low overall } \\
\text { complexity }\end{array}$} \\
\hline Radar & 1 & 1 & 1 & 3 & $\mathbf{0}$ & \\
\hline Transponder & 1 & 1 & 1 & 3 & $\mathbf{0}$ & \\
\hline Power requirements & 1 & 1 & 1 & 3 & $\mathbf{0}$ & \\
\hline Radio communication & 1 & 1 & 1 & 3 & $\mathbf{0}$ & \\
\hline Ground test - Taxi, etc. & 1 & 3 & 3 & 7 & 0.94 & \multirow{5}{*}{$\begin{array}{c}\text { Low-medium } \\
\text { overall } \\
\text { complexity }\end{array}$} \\
\hline Navigation & 1 & 3 & 3 & 7 & 0.94 & \\
\hline Range restrictions & 1 & 3 & 3 & 7 & 0.94 & \\
\hline $\begin{array}{c}\text { Sense and avoid - airspace } \\
\text { availability }\end{array}$ & 1 & 3 & 3 & 7 & 0.94 & \\
\hline Frequency requirements & 1 & 3 & 3 & 7 & 0.94 & \\
\hline $\begin{array}{c}\text { Flight instrumentation and data } \\
\text { recording }\end{array}$ & 2 & 2 & 2 & 6 & $\mathbf{0}$ & \multirow{4}{*}{$\begin{array}{l}\text { Medium overall } \\
\text { complexity }\end{array}$} \\
\hline Chase aircraft & 2 & 2 & 2 & 6 & $\mathbf{0}$ & \\
\hline Laminar flow & 2 & 3 & 3 & 8 & 0.47 & \\
\hline Engine performance & 3 & 3 & 3 & 9 & $\mathbf{0}$ & \\
\hline Environmental control system & 4 & 2 & 2 & 8 & 0.94 & \multirow{12}{*}{$\begin{array}{l}\text { Medium-high } \\
\text { overall } \\
\text { complexity }\end{array}$} \\
\hline Stability and control (RC) & 2 & 3 & 4 & 9 & 0.82 & \\
\hline Simulation - 6-DOF & 3 & 4 & 2 & 9 & 0.82 & \\
\hline Control law development & 4 & 2 & 3 & 9 & $\mathbf{0 . 8 2}$ & \\
\hline Ground Tests - GVT, etc. & 4 & 3 & 3 & 10 & 0.82 & \\
\hline Mission timeline & 2 & 4 & 4 & 10 & 0.94 & \\
\hline Airframe noise & 2 & 4 & 4 & $\mathbf{1 0}$ & 0.94 & \\
\hline Airframe performance & 2 & 4 & 4 & 10 & 0.94 & \\
\hline Control room & 2 & 4 & 4 & 10 & 0.94 & \\
\hline Mission support & 2 & 4 & 4 & 10 & 0.94 & \\
\hline Flight control - Full DFCS & 3 & 4 & 5 & 12 & 0.82 & \\
\hline Engineering workforce & 3 & 5 & 5 & 13 & 0.94 & \\
\hline Flutter & 4 & 5 & 5 & 14 & 0.47 & \multirow{2}{*}{$\begin{array}{l}\text { High overall } \\
\text { complexity }\end{array}$} \\
\hline Airworthiness process & 5 & 5 & 5 & 15 & $\mathbf{0}$ & \\
\hline $\begin{array}{l}\text { Line of sight } \\
\end{array}$ & $\mathbf{0}$ & 3 & 2 & 5 & 1.25 & \multirow{20}{*}{$\begin{array}{l}\text { Largest } \\
\text { deviation in } \\
\text { complexity }\end{array}$} \\
\hline Flight termination system & 0 & 3 & 3 & 6 & 1.41 & \\
\hline Sense and avoid - Pilot $\mathrm{SA}$ & 1 & 2 & 3 & 6 & 1.25 & \\
\hline Stability and control (VR) & 1 & 1 & 4 & 6 & 1.41 & \\
\hline Egress & 4 & 1 & 1 & 6 & 1.41 & \\
\hline Mission management & 1 & 2 & 4 & 7 & 1.25 & \\
\hline Command and control link & $\mathbf{0}$ & 4 & 3 & 7 & 1.70 & \\
\hline Lost link - Mitigation & $\mathbf{0}$ & 4 & 3 & 7 & 1.70 & \\
\hline Cockpit/Ground control station & 1 & 4 & 3 & 8 & 1.25 & \\
\hline Autopilot/Auto throttle/Auto land & 1 & 2 & 5 & 8 & 1.70 & \\
\hline Ground operation & 1 & 3 & 4 & 8 & 1.25 & \\
\hline Flight control - Conventional & 1 & 3 & 4 & 8 & 1.25 & \\
\hline Mission planning & 1 & 3 & 4 & 8 & 1.25 & \\
\hline Pilot workload quantification & 1 & 3 & 5 & 9 & 1.63 & \\
\hline Handling qualities & 1 & 4 & 5 & $\mathbf{1 0}$ & 1.70 & \\
\hline Simulation - HILS & 2 & 3 & 5 & 10 & 1.25 & \\
\hline Envelope expansion & 2 & 4 & 5 & 11 & 1.25 & \\
\hline Pilot display instrumentation & 2 & 4 & 5 & 11 & 1.25 & \\
\hline Flight envelope restrictions & 2 & 4 & 5 & 11 & 1.25 & \\
\hline Operations workforce & 2 & 5 & 5 & 12 & 1.41 & \\
\hline
\end{tabular}


Appendix B: Summarized Results for all Risk Topics

\begin{tabular}{|c|c|c|c|c|c|c|}
\hline Risk & Manned & RPV & Autonomous & Result & St Dev & Result \\
\hline Laminar flow & 1 & 1 & 1 & $\mathbf{3}$ & $\mathbf{0}$ & \multirow{4}{*}{$\begin{array}{c}\text { Low overall } \\
\text { risk }\end{array}$} \\
\hline Power requirements & 1 & 1 & 1 & 3 & $\mathbf{0}$ & \\
\hline Radio Communication & 1 & 1 & 1 & 3 & $\mathbf{0}$ & \\
\hline Transponder & 1 & 1 & 1 & 3 & $\mathbf{0}$ & \\
\hline Flutter & 2 & 1 & 1 & 4 & 0.47 & \multirow{13}{*}{$\begin{array}{c}\text { Low- } \\
\text { medium } \\
\text { overall risk }\end{array}$} \\
\hline Stability and control - Evaluation & 1 & 1 & 3 & 5 & 0.94 & \\
\hline $\begin{array}{c}\text { Egress } \\
\end{array}$ & 3 & 1 & 1 & 5 & 0.94 & \\
\hline Line of sight & 1 & 2 & 2 & 5 & 0.47 & \\
\hline Chase aircraft & 1 & 2 & 2 & 5 & 0.47 & \\
\hline Range restrictions & 1 & 2 & 2 & 5 & 0.47 & \\
\hline Radar & 1 & 2 & 3 & 6 & 0.82 & \\
\hline Mission management & 1 & 2 & 3 & 6 & 0.82 & \\
\hline Frequency requirements & 1 & 3 & 2 & 6 & 0.82 & \\
\hline Navigation & 1 & 3 & 3 & 7 & 0.94 & \\
\hline Pilot display instrumentation & 1 & 3 & 3 & 7 & 0.94 & \\
\hline Flight termination system & 1 & 3 & 3 & 7 & 0.94 & \\
\hline Control law development & 1 & 3 & 3 & 7 & 0.94 & \\
\hline Engineering workforce - Development & 2 & 2 & 2 & 6 & $\mathbf{0}$ & \multirow{11}{*}{$\begin{array}{c}\text { Medium } \\
\text { overall risk }\end{array}$} \\
\hline Simulation - 6-DOF & 2 & 2 & 2 & 6 & $\mathbf{0}$ & \\
\hline $\begin{array}{l}\text { Flight instrumentation and data } \\
\text { recording }\end{array}$ & 2 & 2 & 2 & 6 & $\mathbf{0}$ & \\
\hline $\begin{array}{c}\text { Ground tests } \\
\text { - Preflight, GVT, etc. } \\
\end{array}$ & 2 & 2 & 2 & 6 & $\mathbf{0}$ & \\
\hline Instrumentation ground testing & 3 & 2 & 2 & 6 & $\mathbf{0}$ & \\
\hline $\begin{array}{c}\text { Operations workforce - Physical } \\
\text { Danger }\end{array}$ & 3 & 2 & 2 & 7 & 0.47 & \\
\hline Engineering workforce - Test support & 2 & 3 & 3 & 8 & 0.47 & \\
\hline Simulation - HILS & 2 & 3 & 3 & 8 & 0.47 & \\
\hline Operations workforce - Program & 2 & 3 & 3 & 8 & 0.47 & \\
\hline Environmental control system & 3 & 3 & 3 & 9 & $\mathbf{0}$ & \\
\hline Engine performance & 3 & 3 & 3 & 9 & $\mathbf{0}$ & \\
\hline Airspace availability & 2 & 3 & 4 & 9 & 0.82 & \multirow{7}{*}{$\begin{array}{l}\text { Medium- } \\
\text { high overall } \\
\text { risk }\end{array}$} \\
\hline Stability and control - Development & 2 & 3 & 4 & 9 & 0.82 & \\
\hline Envelope expansion & 2 & 3 & 4 & 9 & 0.82 & \\
\hline Upset recoveries & 2 & 2 & 5 & 9 & 0.82 & \\
\hline Flight control - Full DFCS & 2 & 4 & 4 & 10 & 0.94 & \\
\hline Mission support & 2 & 4 & 4 & 10 & 0.94 & \\
\hline Ground Testing - Taxi, etc. & 2 & 4 & 4 & 10 & 0.94 & \\
\hline Lost link & 1 & 4 & 2 & 7 & 1.25 & \multirow{10}{*}{$\begin{array}{c}\text { Largest } \\
\text { deviation in } \\
\text { risk }\end{array}$} \\
\hline Airframe performance & 2 & 1 & 4 & 7 & 1.25 & \\
\hline Pilot situational awareness & 1 & 3 & 4 & 8 & 1.25 & \\
\hline Command and control link & 1 & 4 & 3 & 8 & 1.25 & \\
\hline Cockpit/Ground control station & 1 & 5 & 3 & 9 & 1.63 & \\
\hline Autopilot/Auto throttle/Auto land & 1 & 4 & 4 & 9 & 1.41 & \\
\hline Airworthiness process & 2 & 3 & 5 & 10 & 1.25 & \\
\hline Flight control - Conventional & 1 & 4 & 5 & 10 & 1.70 & \\
\hline Handling qualities & 1 & 4 & 5 & 10 & 1.70 & \\
\hline Mission Timeline & 1 & 4 & 5 & 10 & 1.70 & \\
\hline
\end{tabular}




\section{Acknowledgments}

The authors acknowledge the support of the CAS program within ARMD and the CAS X-plane Study Design Team leads. Additionally the authors thank all of the support within the team that formulated the major parts of this document at Armstrong Flight Research Center, which included discipline experts in areas of piloting, flight operations, instrumentation, simulation, controls, aerodynamics, safety, and leadership. Specifically we thank Troy Asher, David (Nils) Larson, Scott Howe, Timothy Cox, Marlin Pickett, Gary Cosentino, Steve Sterk, and many others that contributed their time and expertise to the generation of the data contained in this document.

\section{References}

${ }^{1}$ National Aeronautics and Space Administration, "X-planes Could Lead to Substantial Aviation Improvements and Big Economic Benefits," URL: http://www.nasa.gov/feature/x-planes-could-lead-to-substantial-aviation-improvements-and-bigeconomic-benefits [cited 6 April 2017].

2"Recapturing NASA's Aeronautics Flight Research Capabilities," National Academies Press, Washington DC, 2012, pp. 2-3.

${ }^{3}$ Davies, M. B., "Doing a Successful Research Project Using Qualitative or Quantitative Methods," Palgrave Macmillan, New York, 2007.

${ }^{4} 412$ OSS/OSA, "Instruction 13-100, Nuclear, Space, Missile, Command and Control,” EdwardsAFBI 13-100, 2016, Chapter 14.

${ }^{5}$ Range Safety Group Range Commanders Council, "Range Safety Criteria for Unmanned Air Vehicles,” Document 323-99, December 1999.

${ }^{6}$ Sterk, S., "X-1 to X-Wings, Developing a Parametric Cost Model," ICEAA, Atlanta, Georgia, June 7, 2016.

${ }^{7}$ Sterk, S. A., and Olguin, A., "NASA's X-Plane Database and Parametric Cost Model," ICEAA, Bristol, UK, October 17$20,2016$. 